\title{
Estado isostático del abanico del rio Magdalena a partir de la interpretación de anomalías de campos potenciales, Cuenca Colombia
}

\author{
Luis Alejandro Garzón-Moreno ${ }^{1 *}$; Orlando Hernández-Pardo ${ }^{1}$
}

DOI: http://dx.doi.org/10.18273/revbol.v40n2-2018003@ @ (1)

Forma de citar: Garzón-Moreno, L.A., y Hernández-Pardo, O. (2018). Estado isostático del abanico del rio Magdalena a partir de la interpretación de anomalías de campos potenciales, Cuenca Colombia. Boletín de Geología, 40(2), 55-67. DOI: 10.18273/revbol.v40n2-2018003.

\section{RESUMEN}

El propósito principal de esta investigación es esclarecer el estado isostático del área de abanico del Rio Magdalena a partir de la interpretación de anomalías de campos potenciales en la Cuenca Colombia con el fin de profundizar el conocimiento teórico sobre los procesos geodinámicos y el fenómeno de la isostasia. Las investigaciones sugieren que la condición isostática de la corteza está fuertemente afectada por la correlación entre la anomalía gravimétrica de aire libre (FAGA) y los efectos gravimétricos del terreno (TGE).

El análisis e interpretación incluyen el procesamiento y la elaboración de mapas de las anomalías gravimétricas y magnéticas. Las correlaciones e interpretaciones de las anomalías gravimétricas confirman perturbaciones isostáticas a lo largo del margen noroccidental colombiano y la Cuenca Colombia. La validación de los resultados se ha realizado mediante el modelamiento directo e inverso de datos de gravimetría y magnetometría y posterior comparación con modelos de espesor de corteza a partir de datos sísmicos. Los modelos sugieren un bajo ángulo de subducción y la deformación elástica de la placa Caribe, el cual se muestra afectada por la carga sedimentaria del paleo-delta del río Magdalena, las provincias geotectónicas de los cinturones del Sinú - San Jacinto y la corteza continental.

Palabras clave: placa Caribe; gravimetría; magnetometría; isostasia; delta del Magdalena; Cuenca Colombia.

\section{Isostatic state of fan Magdalena river from the interpretation of potential fields anomalies, Colombia Basin}

\begin{abstract}
The main purpose of this research is to clear up the isostatic state of the fan area of the Magdalena River from the interpretation of gravity and magnetic anomalies of the Colombia Basin in order to improve the theoretical knowledge on the geodynamic processes and the phenomenon of isostasy. Previous research suggests that the isostatic condition of the crust is strongly affected by the correlation between Free Air Gravity Anomalies (FAGA) and Terrain Gravity Effects (TGE).

The analysis and interpretation include processing and mapping of gravity and magnetic anomalies. Correlations and interpretations of gravimetric anomalies confirm isostatic perturbations along the Colombian northwestern margin and the Colombia Basin. The validation of the results was done through the forward and inverse modeling from gravity and magnetic data and subsequent comparison with models of crust thickness from seismic data. The models suggest a low subduction angle and elastic deformation of the Caribbean plate, which is affected by the sedimentary load of the paleo-delta of the Magdalena River, the geo-tectonic provinces of the Sinú-San Jacinto belt and the continental crust.
\end{abstract}

Keywords: Caribbean plate; gravity method; magnetic method; isostasy; Magdalena delta; Colombia Basin.

${ }^{1}$ Departamento de Geociencias, Universidad Nacional de Colombia, Bogotá, Colombia. (*) lagarzonm@unal.edu.co, ohernandezp@unal.edu.co. 


\section{INTRODUCCIÓN}

La sedimentación del Rio del Magdalena forma un extenso abanico en el sur de la cuenca Colombia, noroeste de Colombia, formado principalmente por el transporte y acumulación de sedimentos provenientes del Río Magdalena, el cual abarca 1.500 km de longitud, siendo el principal sistema de drenaje en Colombia (FIGURA 1). El abanico se extiende alrededor de $68.000 \mathrm{~km}^{2}$, con un volumen de $180.000 \mathrm{~km}^{3}$ y con una extensión mar adentro de alrededor de 232 km (Kolla et al., 1984).

Previos estudios de la morfología y estratigrafía del abanico del Magdalena muestran fuerte evidencia que, a pesar de su configuración a lo largo de un margen activo (subducción del Caribe), el abanico presenta características similares a los que se encuentran en los grandes sistemas deltaicos de los mayores ríos del mundo a lo largo de márgenes pasivos (Romero-Otero et al, 2015). Además, trabajos previos (Pirmez et al., 1990; Pince et al., 2003), demuestran que hay una relación con los cambios del curso de la desembocadura del Río Magdalena con el tectonismo regional.

El término isostasia es usado para describir la condición por la cual la corteza de la Tierra y el manto tienden a hallarse en equilibrio másico. En una forma más simple, es visualizar la delgada corteza terrestre flotando sobre el denso manto. La geodinámica sobre la superficie terrestre tales como las glaciaciones - desglaciaciones, la acumulación de sedimento, el volcanismo y entre otros, alteran el equilibrio isostático.

La sísmica, magnetometría y gravimetría indican que las capas más externas de la Tierra generalmente se ajustan a estas perturbaciones. Uno de los principales objetivos de los estudios isostáticos desde el pasado siglo $\mathrm{XX}$ ha sido determinar las escalas temporales y espaciales sobre la cual estos ajustes ocurren. Esta información provee contrastes de la naturaleza física de las capas más externas de la Tierra, mejorando nuestro entendimiento de los complejos procesos geodinámicos, tales como el levantamiento de cinturones orogénicos y la formación de cuencas sedimentarias.

El propósito de este estudio es analizar el estado isostático del abanico (también conocido como paleodelta) del Río Magdalena utilizando bases de datos de batimetría, gravimetría y magnetometría de la Cuenca Colombia. De lo anterior se elaboraron mapas de anomalías gravimétricas y magnéticas. Además, con el fin de representar mejor los datos, se realizaron análisis de correlaciones usando índices locales de favorabilidad. Por otro lado, a fin de corroborar las interpretaciones, se efectuaron modelos inversos a partir de las anomalías de aire libre-Bouguer y campo magnético total reducido al polo. Esta interpretación en conjunto con modelos geológicos teóricos desarrollados (Hernandez, 2006) sirven para explicar el resultado de la respuesta de las anomalías gravimetrías y magnéticas de terrenos en equilibrio o desequilibrio isostático.

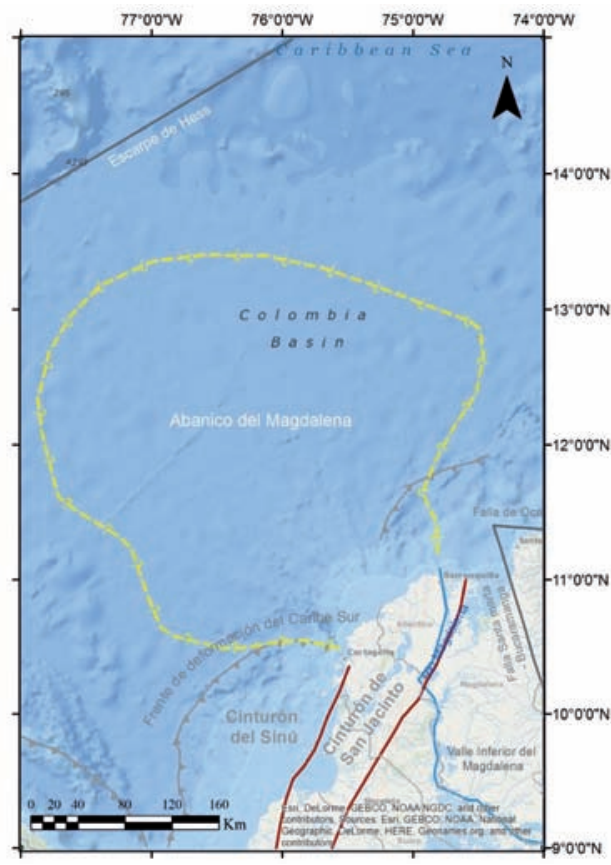

FIGURA 1. Localización del área de estudio. 


\section{METODOLOGÍA}

Con base en las coordenadas geográficas que delimitan el área del abanico del Magdalena se procedió a realizar una serie de mapas que incluyen la cuenca Colombia, los cinturones plegados de Sinú - San Jacinto, la cuenca de Urabá offshore y el valle inferior del Magdalena (Subcuencas San Jorge - El plato), una vez establecidas las coordenadas del área de estudio se procedió a consultar las bases de datos de gravimetría y magnetometría. La base de datos gravimétrica fue obtenida de la compilación de datos del Exploration \& Production Information Service (EPIS) administrada por la Agencia Nacional de Hidrocarburos (ANH), compuesta por 48.673 estaciones, las cuales compilan información del modelo digital del terreno y gravimetría de la Cuenca Colombia. La base de datos magnéticos fue obtenida de la compilación de datos del Geological Survey of Canada (GSC), United States Geological Survey (USGS), y el Consejo de Recursos Minerales de México (CRM). Esta base de datos es reconstruida a partir de la compilación de estudios aerotransportados, como también de datos marinos de la National Geophysical Data Center of the National Oceanic and Atmospheric Administration.

A partir de estas bases de datos se generaron procesos de interpolación y generación de grillas de campos temáticos de gravimetría y magnetometría, con despliegue de colores en formatos raster, incluyendo barras explicativas de colores, contornos de isovalores y leyendas explicativas.

Los mapas temáticos de anomalías gravimétricas y magnéticas determinaron las tendencias de las anomalías con respecto a la morfología, geología, e infiriendo los posibles cuerpos causantes de las anomalías y cómo estos responden al fenómeno de la isostasia.

\section{MARCO TEÓRICO}

\section{Compensación isostática}

El estado de la compensación isostática de una región puede ser aproximada, haciendo uso de las anomalías gravimétricas (Kearey et al., 2009). La anomalía isostática, $g_{I A}$, es definida como la anomalía de Bouguer menos la anomalía gravimétrica del subsuelo de compensación. Así, la anomalía de Bouguer, $g_{S B A}$, se relaciona con la anomalía de aire libre $g_{F A G A}$, como:

$$
g_{S B A}=g_{F A G A}-g_{B}
$$

De este modo para una compensación de acuerdo Airy, está dada por:

$$
g_{I A}=g_{S B A}-A_{\text {root }}
$$

Donde $A_{\text {root }}$ es la anomalía de la raíz de compensación. Esta anomalía puede ser calculada como $2 \pi G\left(\rho_{c}-\rho_{m}\right) b$. Combinando las ecuaciones, se obtiene que:

$$
g_{I A}=g_{F A G A}-2 \pi G \rho_{c} h-2 \pi G\left(\rho_{c}-\rho_{m}\right) \frac{h \rho_{c}}{\rho_{m}-\rho_{c}}
$$

Dónde $G$ es la constante gravitacional, $h$ la altura del cuerpo sobre el nivel del mar, $\rho_{c}$ la densidad de la corteza y $\rho_{m}$ la densidad del manto.

$$
g_{I A}=g_{F A G A}
$$

Se demuestra que la anomalía isostática es igual a la anomalía de aire libre, de este modo se representa que la interpretación de la anomalía gravimétrica de aire libre es un método simple para determinar el estado isostático o grado de compensación isostático de un terreno.

Por otro lado, la anomalía de aire libre (FAGA) al ser igual a la anomalía isostática permite determinar aspectos isostáticos de un terreno, los cuales están asociados a los efectos gravimétricos del terreno (TGE) y los efectos gravimétricos de la "raíz" cortical RGE (o anomalía gravimétrica de Bouger CBA), esta relación puede expresarse como:

$$
g_{F A G A}=g_{T G E}+g_{C B A}
$$

De acuerdo a esta relación, el equilibrio isostático de un terreno es alcanzado, cuando la anomalía de aire libre es igual a cero. Lo cual implica que TGE y CBA son de igual magnitud, pero de signo contrario (FIGURA 2). La correlación numérica entre FAGA y TGE, permite cuantificar el grado de compensación isostático de un terreno (Hernandez, 2006; Hinze et al., 2013; von Frese et al., 1997). 

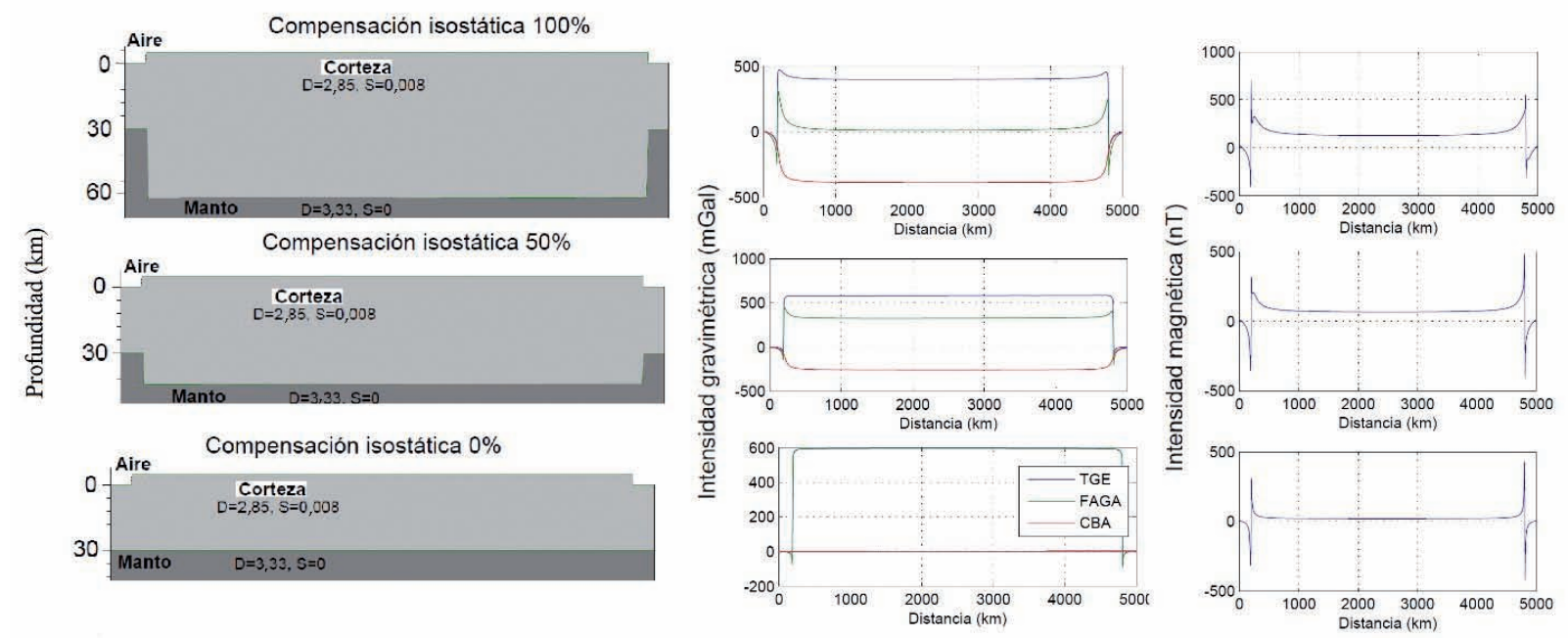

FIGURA 2. Modelo geológico simple de compensación isostática de acuerdo al modelo de Airy para una carga topográfica con altura de $5 \mathrm{~km}$, con respuesta gravimétrica y magnética para diferentes grados de compensación isostática.

\section{Coeficientes de correlación}

Se calcularon los coeficientes de correlación CC(k) para analizar las anomalías gravimétricas co-registradas. Por lo que se determinó la transformada de Fourier de dos señales gravimétricas $\chi(k)$ y $\gamma(k)$ en coordenadas de número de onda común.

$$
C C(k)=\cos \left(\Delta \vartheta_{k}\right)=\frac{\chi(k)^{*} \gamma(k)}{|\chi(k)||\gamma(k)|}
$$

Donde el numerador es el producto punto entre los vectores de onda con diferencia de fase $\Delta \theta_{k}=\left[\theta_{\gamma(k)}-\theta_{\chi(k)}\right]$ (von Frese et al., 1997). En otras palabras, el coeficiente de correlación entre dos vectores de onda es su producto punto normalizado, el cual es simplificado por el coseno de la diferencia de fase de las dos señales.

\section{Análisis de anomalías correlacionadas}

Para facilitar la interpretación gráfica entre las correlaciones, los datos fueron estandarizados en coeficientes adimensionales:

$$
S_{i}=\frac{b_{i}-\mu_{B}}{\sigma_{B}}
$$

Donde $\mu_{B}, \sigma_{B}$ y $b_{i}$ son el promedio, desviación estándar y valor de la estación respectivamente. Posteriormente los coeficientes estandarizados fueron transformados en coeficientes normalizados (von Frese et al., 1997).

$$
n_{i}(B)=S_{i}^{*} \sigma_{N}-\mu_{N}
$$

Con el fin de identificar correlaciones en las señales, se aplicaron índices locales de favorabilidad (Merriam y Sneathh, 1966). Las anomalías directamente correlacionadas fueron mapeadas por la expresión SLFI.

$$
S L F I_{i}=n_{i}(B)+n_{i}(D)
$$

Los coeficientes $S L F I_{i}>0$ enfatizan las características correlacionadas positivas (correlación peak to peak), mientras los coeficientes $S L F I_{i}<0$ enfatizan las características correlacionadas negativas (correlación trough to trough).

Las anomalías inversamente correlacionadas fueron mapeadas por la expresión DLFI

$$
D L F I_{i}=n_{i}(B)-n_{i}(D)
$$

Los coeficientes $D L F I_{i}>0$ enfatizan las características positivas de $\mathrm{B}$ correlacionadas con las características negativas de D (correlación peak to trough), mientras los coeficientes $D L F I_{i}<0$ mapean las características negativas de $\mathrm{B}$ correlacionadas con las características positivas de D (correlación trough to peak).

\section{Teorema de Poisson}

El teorema de Poisson ofrece la base para correlacionar los efectos gravimétricos y magnéticos de una fuente en común. Para un análisis de correlación, las fases de las anomalías deben ser la misma de acuerdo a la relación de Poisson, la cual es alcanzada reduciendo las anomalías del campo magnético total al polo DRTP (TMFA) y por el otro lado tomando la primera derivada vertical de la anomalía gravimétrica FVD (FAGA) (FIGURA 3). 


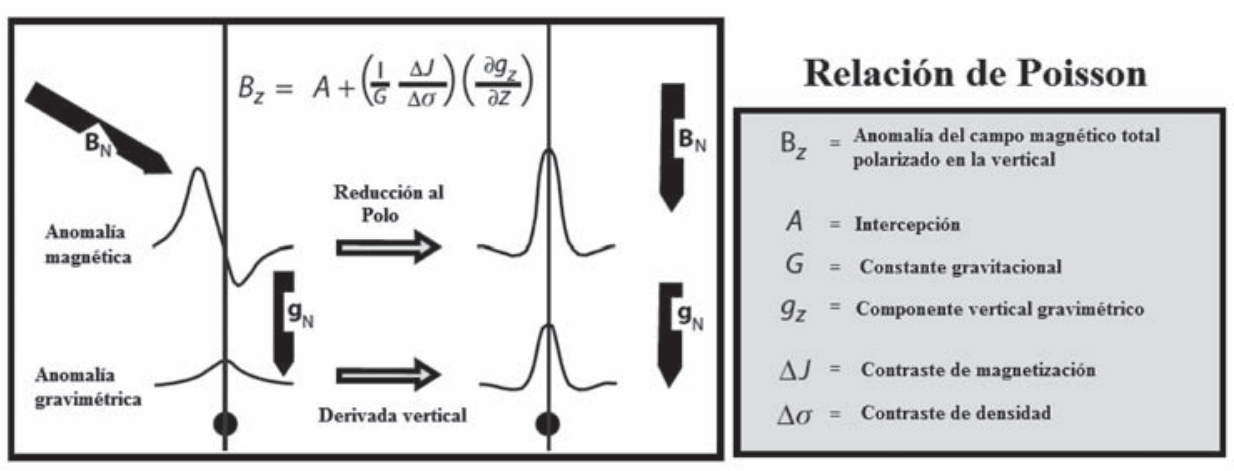

FIGURA 3. Relación de Poisson entre anomalías magnéticas y gravimétricas para una fuente en común con densidad positiva y magnetización contrastante (modificado de Hinze et al., 2013).

\section{RESULTADOS}

\section{Modelo digital del terreno (DTM)}

Las sub-cuencas sedimentarias el Plato y San Jorge del valle inferior del Magdalena presentan relieves variados, siendo más bajo en la sub-cuenca San Jorge. Los cerros Tubará, Luruaco, Turbaco, San Onofre, San Antero y las Serranías Abibe - Las Palomas y San Jacinto, resaltan con respecto al nivel del mar con alturas por encima de los $200 \mathrm{~m}$. El mayor rasgo morfológico es caracterizado por la Sierra Nevada de Santa Marta (SNSM) con alturas por encima de los $5.000 \mathrm{~m}$. El modelo digital del terreno (FIGURA 4) varía entre los $-4.330 \mathrm{~m}$ a $769 \mathrm{~m}$, con un valor promedio de $-2.585 \mathrm{~m}$ y una desviación estándar de $1.531 \mathrm{~m}$. El modelo digital del terreno permite estimar los efectos del terreno asociados a la topografía y batimetría de unidades morfológicas. La mayor influencia por efectos del terreno la presentan las sub-cuencas del Plato y San Jorge por la vecindad de la SNSM. La batimetría de la cuenca Sinú en el Mar Caribe muestra una profundización hacia mar adentro con una plataforma continental con extensión reducida y marcada por la abrupta pendiente del talud continental. Sin embargo, este contraste es contrarrestado por la morfología suavizada y arqueada del abanico del Río Magdalena. A demás se resalta el contraste estructural del escarpe de Hess, la estrecha cuenca del golfo de Urabá y la morfología de montes submarinos por debajo de los $-3.000 \mathrm{~m}$.

\section{Anomalía gravimétrica de aire libre (FAGA)}

La anomalía de aire libre (FIGURA 5A) muestra valores mínimos y máximos entre -168 y 208 mGals, con un valor promedio de -13,54 mGals y desviación estándar de 42,56 mGals. Se destaca la delimitación de anomalías negativas de aire libre entre las subcuencas Plato, San Jorge y las subcuencas offshore del Tayrona y del Urabá, en las cuales los valores negativos resaltan la sobrecompensación isostática de estas subcuencas.
Las anomalías de aire libre positivas resaltan la descompensación de la SNSM, el cual alcanza anomalías por encima de los $200 \mathrm{mGals}$. Los cerros desde Tubará y San Antero, y la serranía de San Jacinto de igual manera exhiben anomalías positivas por encima de los $30 \mathrm{mGals}$ resaltando la descompensación del área, aunque los valores presentan una compensación isostática parcial, los valores aún están lejanos de una anomalía de aire libre cercana a cero. El abanico del Magdalena, aunque no exhibe anomalías contrastantes, presenta anomalías negativas entre -20 y -30 mGals, acompañada con anomalías puntuales contrastantes positivas y negativas, posiblemente enlazadas a la geometría del basamento infrayaciente al abanico. En el interior de la Cuenca Colombia, resalta la delimitación de anomalías positivas entre los $20 \mathrm{mGals}$ y $30 \mathrm{mGals}$, exhibiendo una extensa área descompensada isostáticamente, posiblemente asociado con la formación de un abultamiento resultante de la deflexión elástica de la placa Caribe o adelgazamiento de la corteza oceánica.

\section{Efectos gravimétricos del terreno (TGE)}

Los efectos gravimétricos del terreno (FIGURA 5B) se obtuvieron a partir de la diferencia entre las anomalías observadas de aire libre y Bouguer total: $g_{T G E}=g_{F A G A}-g_{C B A}$. Los efectos gravimétricos del terreno varían entre -285 mGals a 78 mGals, con un valor promedio de -171 mGals y desviación estándar de 100,7 mGals. Estos valores están directamente correlacionados al modelo digital del terreno. A mayor elevación es mayor el efecto gravimétrico del terreno y a menor elevación los efectos serán menores. Se resalta los valores positivos de la SNSM, y de los relieves variables de las serranías de San Jacinto, Abibe - Las Palomas, la cuenca del valle inferior del Magdalena y los cerros de Tubará, Luruaco, Turbaco, San Antero y San Onofre. Los valores negativos de los efectos gravimétricos del terreno resultan próximos por debajo del nivel del mar, siendo más negativos al profundizarse en el fondo marino. 


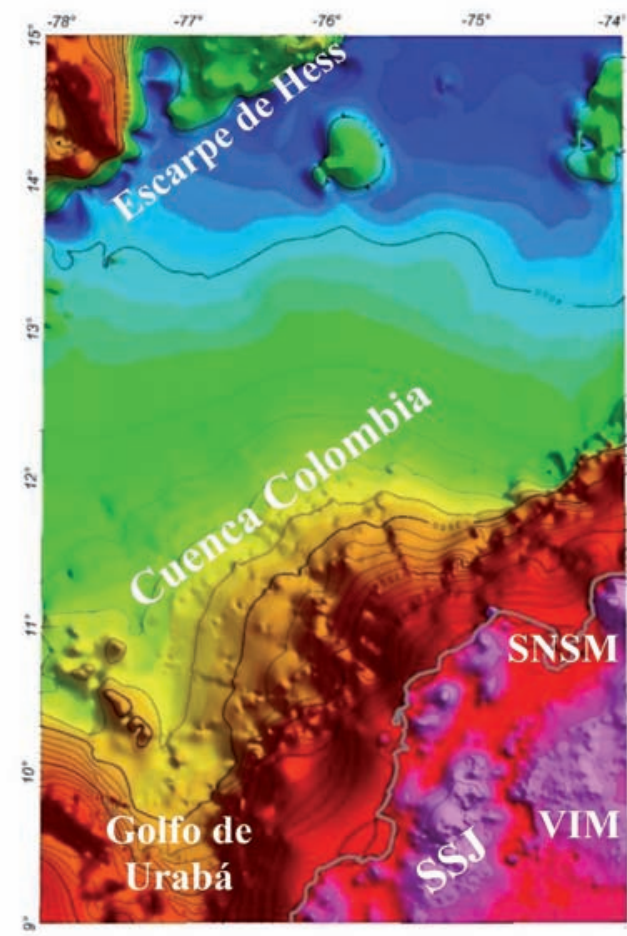

$A R=|-4330 ; 769,3| ; A M=-2585 ; D S=1531$

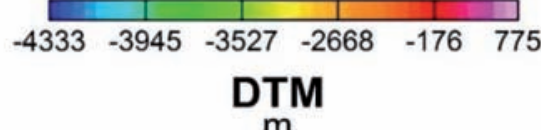

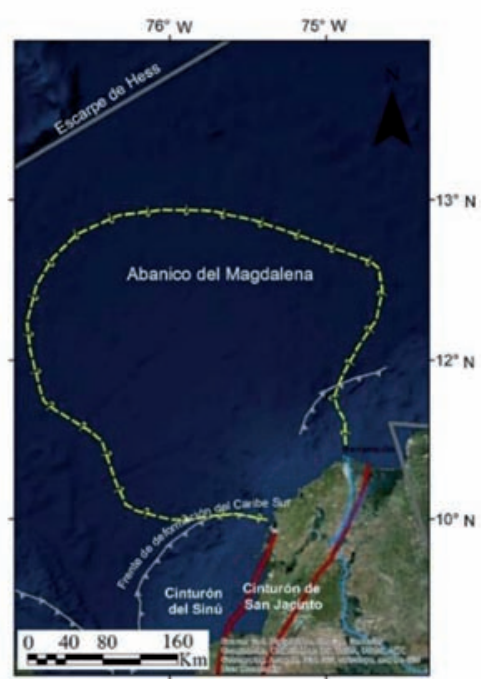

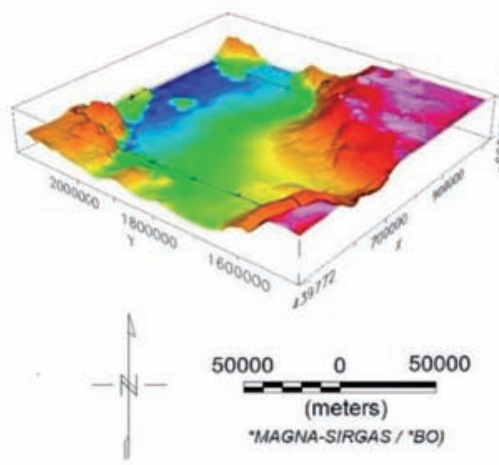

FIGURA 4. Modelo digital del terreno. SSJ: Serranía de San Jacinto; SNSM: Sierra Nevada de Santa Marta; VIM: Valle Inferior del Magdalena. AR: Rango de amplitud; AM: Amplitud promedio; DS: Desviación Estándar.

\section{Anomalía gravimétrica de Bouguer total (CBA)}

La anomalía de Bouguer total (FIGURA 5C) muestra valores entre -285 mGals a $78 \mathrm{mGals,}$ con un valor promedio de 157 mGals y desviación estándar de 105 mGals. La anomalía de Bouguer ha sido obtenida utilizando un valor de densidad de $2,67 \mathrm{~g} / \mathrm{cm}^{3}$. La anomalía de Bouguer resulta siendo los efectos gravimétricos de raíz cortical para un modelo de compensación isostático Airy o, en otras palabras, los efectos del relieve de la interface entre manto y corteza. Se resalta la delimitación de las anomalías negativas para el área continental, resultante de la deficiencia de masa característica de la corteza continental, respecto a la corteza oceánica. Los valores más negativos describen una profundización cortical de las subcuencas Plato y San Jorge. Opuesto a estos, la SNSM exhibe anomalías positivas, apoyando aún más la tesis de que este terreno, esta descompensado isostáticamente. En el interior de la Cuenca Colombia, se resalta la delimitación de anomalías positivas asociadas a un exceso de masa, posiblemente como consecuencia de la formación de un abultamiento resultante de la deflexión elástica de la placa Caribe.

\section{Anomalía del campo magnético total (TMFA)}

Los valores del mapa residual magnético total del campo magnético de la Tierra, son el resultado del valor del campo magnético total menos el IGRF, el cual es un campo magnético regional de longitud de onda larga (FIGURA 6A). El mapa muestra valores entre $-547,9 \mathrm{nT}$ a $548 \mathrm{nT}$, con un valor promedio de - $63 \mathrm{nT}$ y una desviación estándar de $95 \mathrm{nT}$. Debido a la naturaleza bipolar de las anomalías, la interpretación no ha sido implementada.

\section{Anomalía del campo magnético total reducido al polo DRTP (TMFA)}

Con el fin de adecuar los datos magnéticos en la latitud promedio de la zona de estudio. Se realizó un filtrado espectral para reducir los datos al polo magnético, donde la magnetización se observa vertical, de tal manera que simplifica las anomalías observadas. La reducción al polo del campo magnético total muestra valores entre $-895 \mathrm{nT}$ a $715 \mathrm{nT}$, con valor promedio de $-62 \mathrm{nT}$ y una desviación estándar de $131 \mathrm{nT}$. 
La mayoría de las anomalías localizadas en la Cuenca Colombia presentan una distribución heterogénea sin una tendencia evidente preferencial. Sin embargo, sobresalen en general algunas anomalías positivas (FIGURA 6B). Suponiendo una corteza oceánica homogénea con una susceptibilidad magnética en promedio constante, estas anomalías magnéticas positivas estarían relacionadas a engrosamientos de la corteza, debido a irregularidades de la placa Caribe.

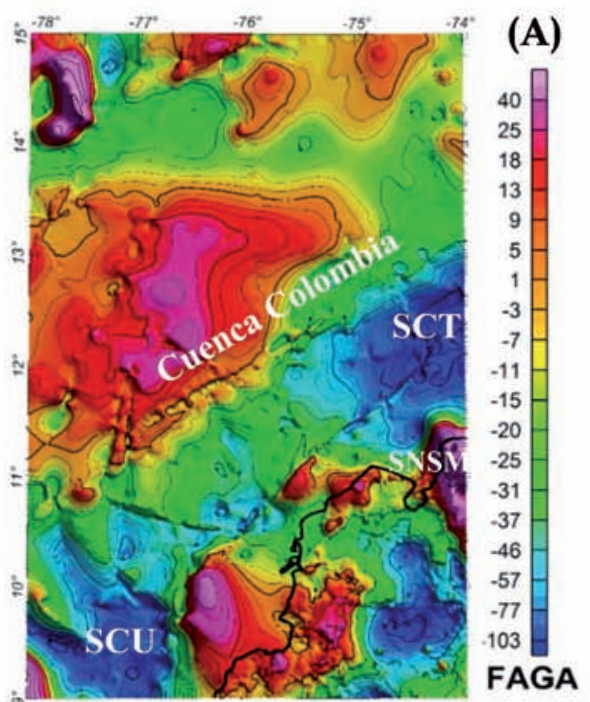

$A R=[-167,9 ; 207,8] ; A M=-13,54 ; D S=42,56$

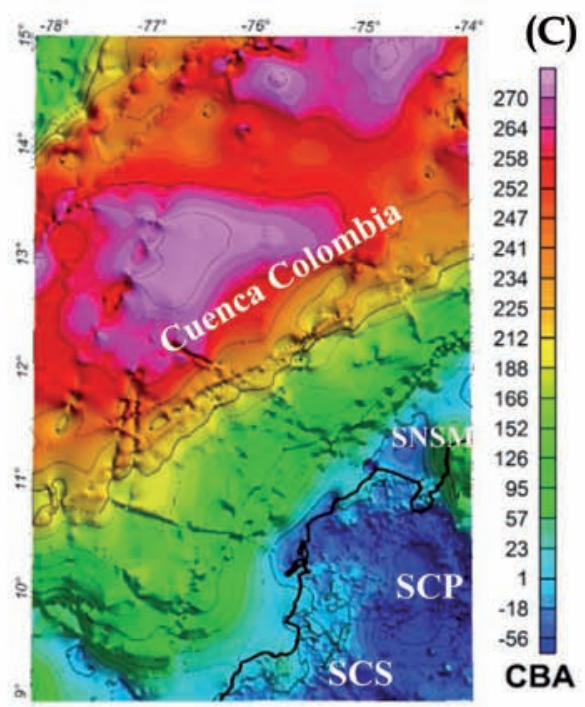

$A R=[-153,1 ; 288,9] ; A M=157,5 ; D S=105,5$

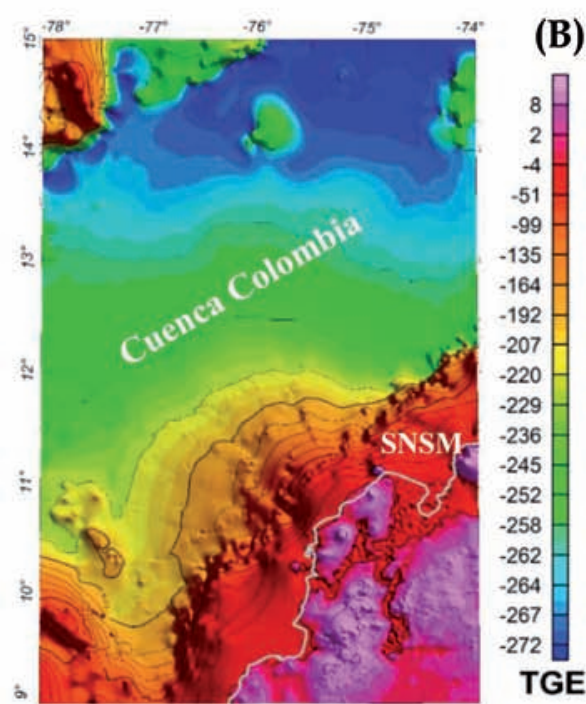

$A R=[-284,9 ; 78,64] ; A M=-171 ; D S=100,7$

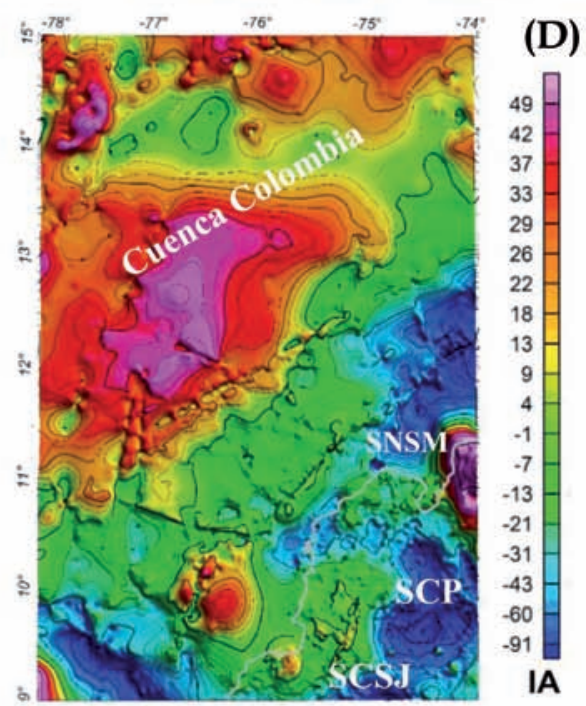

$A R=[-151,7 ; 169,1] ; A M=3,22 ; D S=39,53$
(B)

D)

FIGURA 5. Anomalías gravimétricas. IA: Corresponde a la anomalía isostática modelada de acuerdo al modelo isostático de Airy con una corteza de $30 \mathrm{~km}$ y una densidad de contraste de $350 \mathrm{~kg} / \mathrm{m}^{3}$. AR: Rango de amplitud; AM: Amplitud promedio; DS: Desviación Estándar; SNSM: Sierra Nevada de Santa Marta; SCU: Subcuenca Urabá; SCT: Subcuenca Tayrona; SCP: Subcuenca Plato; SCSJ: Subcuenca San Jorge. 


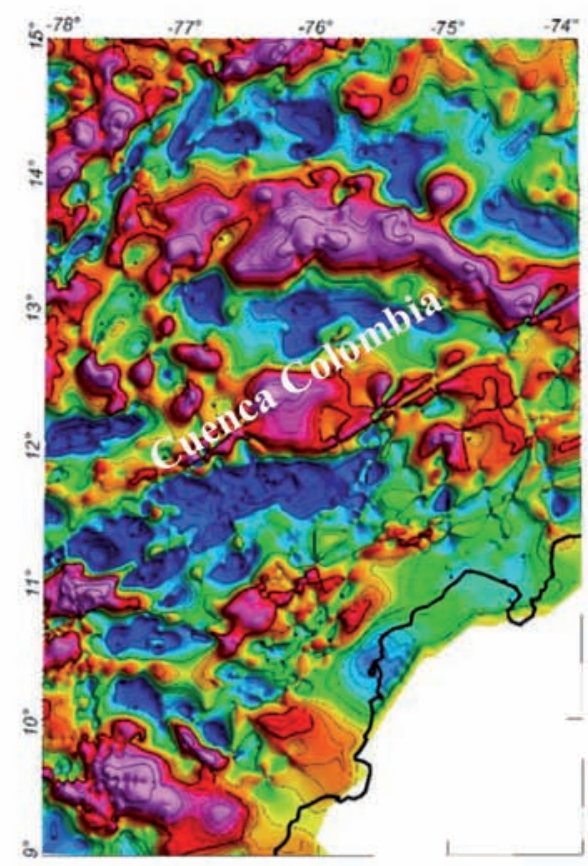

$A R=[-547,9 ; 548] ; A M=63,24 ; D S=95,5$
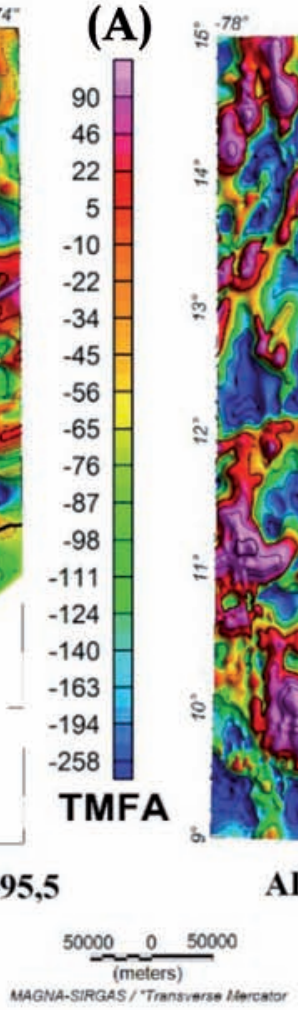

(B)

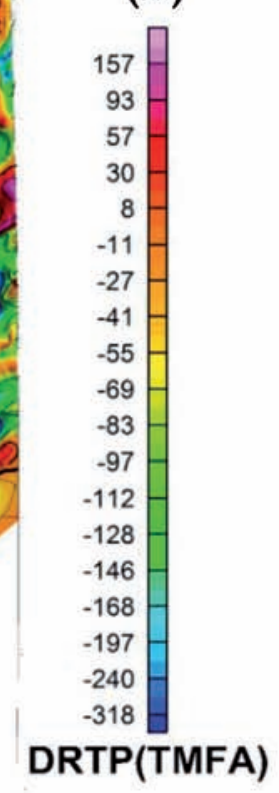

$\mathrm{AR}=[-895,2 ; 715,6] ; \mathrm{AM}=-62,23 ; \mathrm{DS}=131,3$

FIGURA 6. Anomalías magnéticas. AR: Rango de amplitud; AM: Amplitud promedio; DS: Desviación Estándar.

\section{Correlación de la anomalía de aire libre (FAGA) y los efectos gravimétricos del terreno (TGE)}

La correlación entre FAGA y TGE se llevó a cabo usando índices locales de favorabilidad con (SLFI > 1,3922) en la FIGURA 7. Las zonas con amplitudes altas se exhiben principalmente sobre el continente, sobre topografías positivas. La SNSM muestra la máxima correlación de la zona, indicando una fuerte descompensación isostática.

Las amplitudes de correlación intermedias se exhiben a lo largo de la serranía de San Jacinto, en los cerros Tubará - San Onofre y a un fuerte contraste al noroeste del golfo de Morrosquillo, de igual manera asociadas a descompensación isostática.

Para los SLFI $<-1,3922$ muestra una correlación trough to trough intermedia entre FAGA y TGE respectivamente. Esta correlación claramente marca las subcuencas Tayrona y Urabá, las cuales demuestran ser áreas sobrecompensadas isostáticamente. Estas áreas están bajo una intensa exploración de gas e hidrocarburo, asociados con depósitos deltaicos de los ríos Magdalena, Sinú y Atrato, los cuales representan los sistemas de drenaje con mayor aporte de sedimentación del mar Caribe. Por lo tanto, los SLFI de las anomalías correlacionadas pueden marcar fuertemente área de prospección para localizar cuencas sedimentarias (Hernandez, 2006).

Para los DLFI $>1,2703$ muestra la correlación de las altas amplitudes de FAGA y bajas de TGE. A partir de esta correlación se puede marcar las depresiones topográficas sobrecompensadas por cuerpos locales densos (Hernandez, 2006), el cual se evidencia a lo largo del interior de la Cuenca Colombia y la Sierra Nevada de Santa Marta. Además, permite estimar el límite tectónico de las placas Caribe-suramericana. Las correlaciones con DLFI $<-1,2703$, por el otro lado muestra la distribución de zonas de baja densidad y contrastes topográficos positivos. Los DLFI negativos marca la cuenca sedimentaria del valle inferior del Magdalena, permitiendo cartografiar las subcuencas de El Plato y San Jorge. De este modo, los DLFI negativos permite inferir y delimitar cuencas sedimentarias para la exploración de gas e hidrocarburo (Hernandez, 2006). 


\section{Correlación de las anomalías DRTP (TMFA) y FVD (FAGA)}

Para mejorar la interpretación entre DRTP (TMFA) y FVD (FAGA) se aplicaron índices locales de favorabilidad. Las anomalías positivas de DRTP sugieren un variado aumento de la magnetización de la placa Caribe, sin ninguna tendencia preferencial. De igual manera las anomalías negativas implican un decrecimiento de la magnetización.

Las anomalías positivas de FVD (FAGA) sugieren aumento en la densidad de la corteza en la SNSM, cerros y serranías costeras del caribe colombiano, también se muestra una variada distribución de la densidad en la Cuenca Colombia, asociada a cuerpos locales. De igual manera las anomalías negativas, indican bajas densidades de la corteza. Estas anomalías negativas se perciben en las áreas de las subcuencas Tayrona, El Plato y Urabá.

La correlación FVD (FAGA)-peak a DRTP (TFMA)peak (FIGURA 7) sugiere altos contrastes de densidad y magnetización principalmente asociados a la Sierra Nevada de Santa Marta. Por otro lado, las correlaciones intermedias están relacionada con el escarpe de Hess.

La correlación trough to trough muestra la baja densidad y magnetización de la corteza en las subcuencas Tayrona y Urabá como también una variada distribución a lo largo de la placa Caribe. La baja densidad y magnetización pueden resultar de fuentes tales como cuencas sedimentarias, la profundidad de la fosa oceánica, y la posible presencia de intrusiones graníticas con baja densidad y magnetización que las rocas máficas huésped (Hernandez, 2006).

La correlación FVD (FAGA)-peak a DRTP (TFMA)trough muestran las áreas con aumento de densidad y baja magnetización. Estas fuentes están relacionadas con un aumento en el flujo de calor de la corteza y adelgazamiento de la corteza anómalo (Hernandez, 2006).

La correlación FVD (FAGA)-trough a DRTP (TFMA)peak muestra baja densidad y alta magnetización de la corteza. Sin embargo, en el área de estudio es poca o casi nula la correlación, por tal motivo no se sugiere algún arreglo tectónico.

\section{Coeficiente de correlación}

Los filtros de correlación proveen indicios del estado isostático de la litosfera a partir de la correlación entre la anomalía gravimétrica y topográfica regional (Watts, 2001). De acuerdo a Hinze et al. (2013) la correlación entre FAGA y TGE puede reflejar la compensación isostática de la litosfera. Si la correlación es 1 (Total descompensación) o -1 (Total sobrecompensación) entre FAGA y TGE es un terreno con $0 \%$ de compensación isostática, mientras si la correlación es 0 es entonces un $100 \%$ de compensación isostática.

Se calcularon los coeficientes de correlación espectral para analizar las anomalías gravimétricas coregistradas. Por lo que se determinó la transformada de Fourier de dos señales gravimétricas $\chi(k)$ y $\gamma(k)$ en coordenadas de número de onda común, de acuerdo a la ecuación 6. Los coeficientes de correlación entre FAGA, TGE, IA y CBA para el área sobre el abanico del Río Magdalena son mostrados en la TABLA 1. El coeficiente de correlación entre FAGA y TGE muestra una correlación débil de CC $=-0,2548$ indicando la débil - moderada sobrecompensación isostática del abanico del Río Magdalena. La correlación entre IA y TGE muestra una correlación moderada indicando una mayor sobrecompensación respecto a FAGA con un $\mathrm{CC}=-0,5168$.

Los coeficientes de correlación permiten observar la relación inversamente proporcional entre TGE y CBA (RGE). El cual está asociado a los efectos de la topografía y como estos están compensados con los efectos gravimétricos de la raíz cortical.

TABLA 1. Coeficientes de correlación (CC) entre las anomalías gravimétricas para el área de $10,5^{\circ}-13^{\circ}$ latitud y $-74,5^{\circ}--77^{\circ}$ longitud.

\begin{tabular}{ccccc}
\hline CC & FAGA & TGE & IA & $\begin{array}{c}\text { CBA } \\
\text { (RGE) }\end{array}$ \\
\hline FAGA & 1 & $-0,2548$ & $-0,9477$ & 0,7221 \\
TGE & $-0,2548$ & 1 & $-0,5168$ & $-0,9188$ \\
IA & 0,9477 & $-0,5168$ & 1 & 0,8431 \\
CBA & 0,7021 & $-0,9188$ & 0,8431 & 1 \\
(RGE) & & & & \\
\hline
\end{tabular}



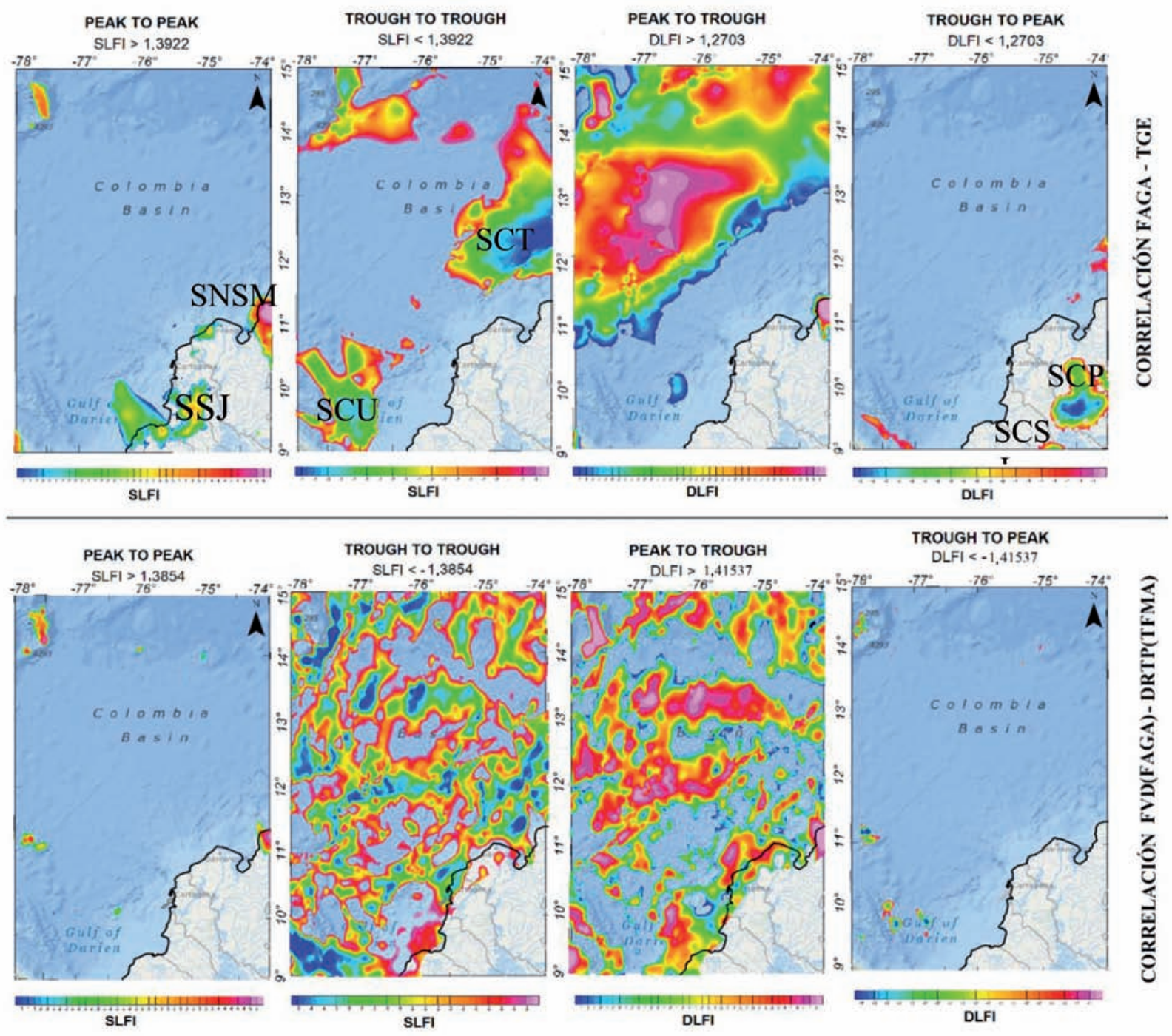

FIGURA 7. Suma de índices locales de favorabilidad (SLFI) y Diferencia de índices locales de favorabilidad (DLFI) SSJ: Serranía de San Jacinto; SNSM: Sierra Nevada de Santa Marta; SCU: Subcuenca Urabá; SCT: Subcuenca Tayrona; SCP: Subcuenca Plato; SCSJ: Subcuenca San Jorge.

\section{Profundidad del MOHO y espesor de la corteza}

La discontinuidad de Mohorovic (FIGURA 8) fue obtenida a partir del modelo digital del terreno y la anomalía de Bouguer total, con una compensación isostática respecto al nivel del mar de $30 \mathrm{~km}$, una densidad de contraste para la raíz cortical de $0,35 \mathrm{~g} /$ $\mathrm{cm}^{3}$, y una densidad topográfica de $2,67 \mathrm{~g} / \mathrm{cm}^{3}$. Se presentan valores de profundidad del $\mathrm{MOHO}$ entre $-35.830 \mathrm{~m}$ a $-9.392 \mathrm{~m}$, con una profundidad promedio de -17.760 m y desviación estándar de 7.356 m. Los valores altos corresponden para la corteza oceánica con una profundidad entre $-11.000 \mathrm{~m}$ a $-9.000 \mathrm{~m}$ y los bajos valores a la corteza continental están entre $-36.000 \mathrm{~m}$ a $-30.000 \mathrm{~m}$.

El espesor de la corteza (FIGURA 8) se obtuvo de la suma entre la profundidad del MOHO y el modelo digital del terreno. Con un espesor de corteza entre 5.060 $\mathrm{m}$ a $36.610 \mathrm{~m}$, con un espesor promedio de $15.170 \mathrm{~m}$ y desviación estándar de $8.888 \mathrm{~m}$. De acuerdo al modelo se resalta la delgada corteza oceánica con respecto al espesor de la corteza continental. 


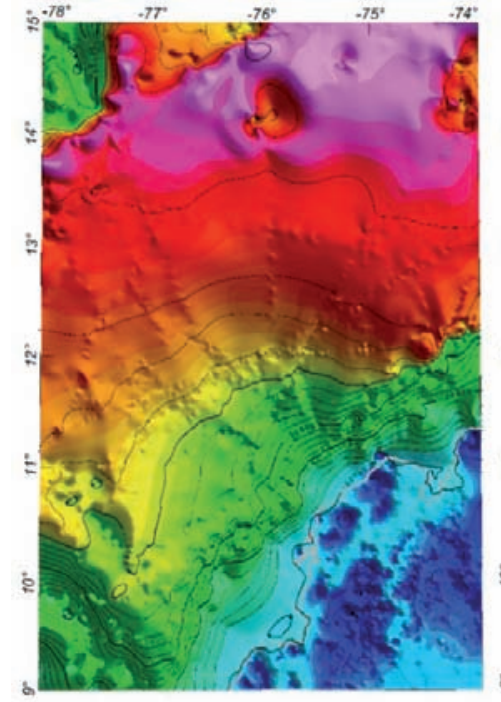

$A R=|-35830 ;-9392| ; A M=-17760 ; D S=7356$

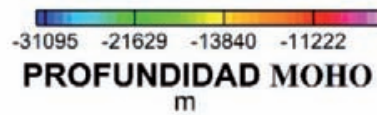

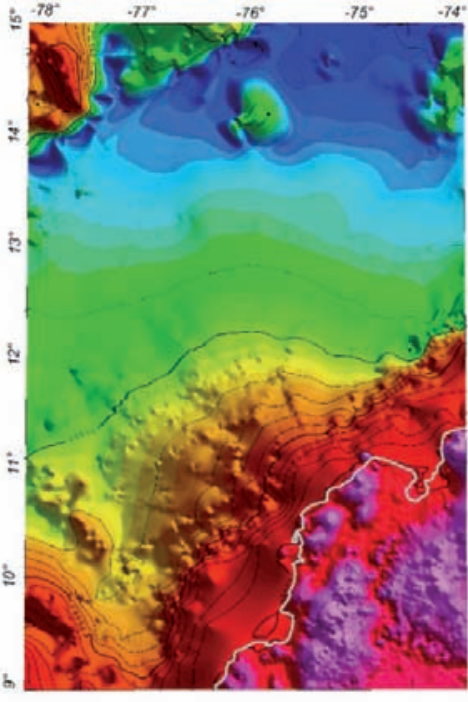

$A R=|5060 ; 36610| ; A M=15170 ; D S=8888$

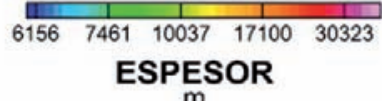

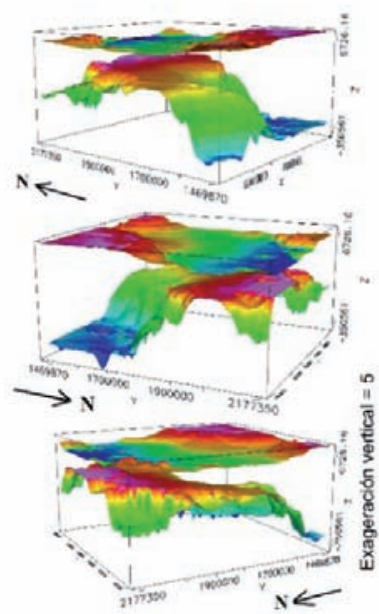

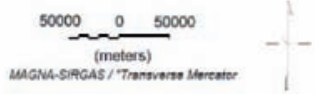

FIGURA 8. Profundidad del MOHO, con una profundidad de compensación de $30 \mathrm{~km}$ y una densidad de contraste de $350 \mathrm{~kg} / \mathrm{m}^{3}$. El espesor de la corteza corresponde a la diferencies entre el modelo digital del terreno y la profundidad del MOHO. AR: Rango de amplitud; AM: Amplitud promedio; DS: Desviación Estándar.

\section{Sección transversal de la corteza a partir de modelamiento inverso}

La FIGURA 9 muestra una sección transversal interpretada a partir de las anomalías gravimétricas y magnéticas en dirección NW - SE a lo largo de la subcuenca El Plato y el abanico del Río Magdalena hasta mar adentro. El modelo igualmente sugiere un ángulo de subducción $\sim 10^{\circ}$ para la placa Caribe con un espesor promedio de $10 \mathrm{~km}$. La corteza continental debajo de la subcuenca El Plato alcanza un espesor de $25 \mathrm{~km}$ adelgazándose en dirección NW.
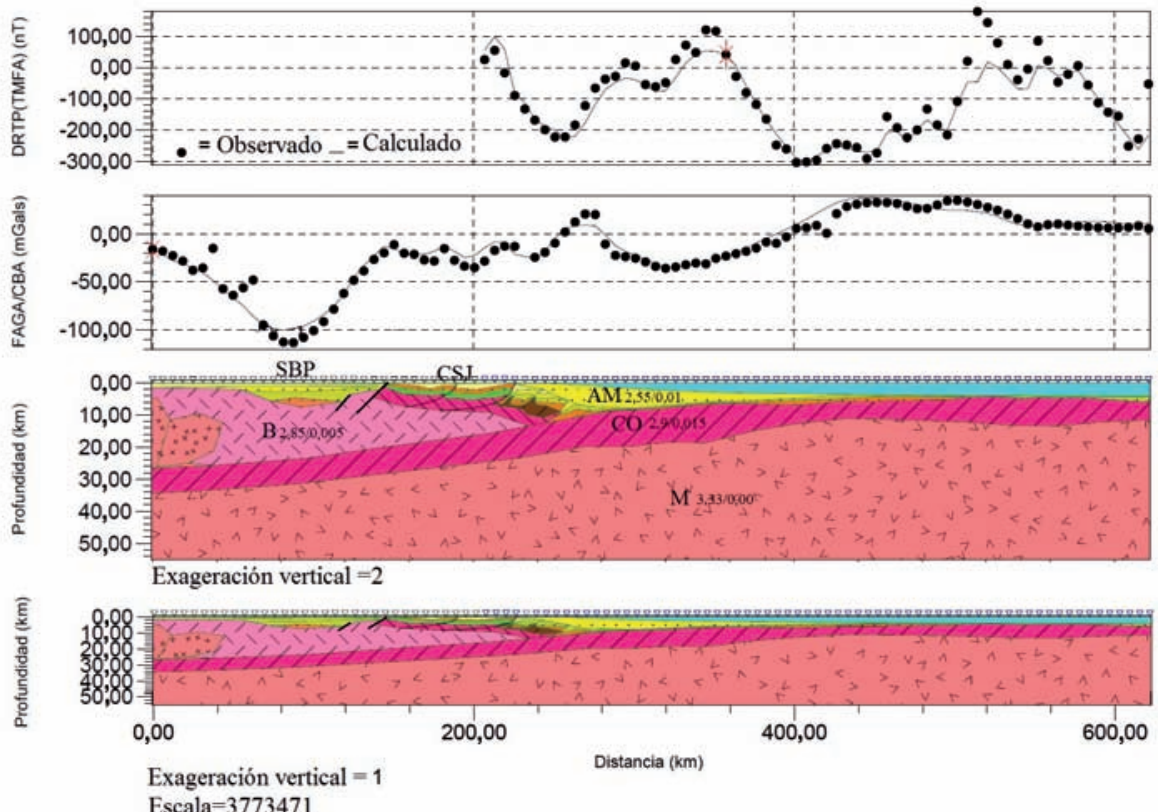

FIGURA 9. Modelo gravimétrico y magnético 2D. AM: Abanico del Río Magdalena; CO: Corteza Oceánica (Placa Caribe); B: Basamento continental; M: Manto; SBP: Subcuenca Plato; CSJ: Cinturón plegador de San Jacinto. 
El fuerte contraste de la anomalía positiva gravimétrica y negativa magnética sugieren un adelgazamiento de la placa Caribe en el interior de la Cuenca Colombia, seguido de un engrosamiento el cual puede estar relacionado con el engrosamiento del modelo anterior.

La respuesta de la anomalía negativa de FAGA del prodelta del Río sugiere la deficiencia de masa, indicando descompensación isostática. Sin embargo, el frente deltaico y la llanura deltaica en respuesta a los efectos gravímetros del terreno elevan la anomalía de aire libre, indicando una compensación isostática parcial.

Las anomalías gravimétricas negativas resultantes de la subcuenca El Plato, permiten modelar una cuenca con profundidad y espesor de sedimentos entre los 6 y $8 \mathrm{~km}$.

\section{CONCLUSIONES}

Los modelos realizados por modelamiento directo y la inversión de FAGA para una litosfera isostáticamente diferenciada puede ser implementada en la realización de modelos isostáticos. Siendo FAGA la respuesta gravimétrica que provee información sobre el estado isostático de la litosfera.

La evaluación de los mapas gravimétricos del área de estudio permite reconocer estructuras geológicas con diferentes estados isostáticos en el noroccidente del caribe colombiano. Esta condición es cambiante a lo largo y ancho del límite tectónico compresivo de las placas Caribe-Suramericana. Las anomalías negativas de FAGA están asociadas a las subcuencas sedimentarias de Tayrona, Urabá, Plato y San Jorge. De acuerdo a la evaluación de FAGA, estas subcuencas exhiben sobrecompensación isostática, causada por la deficiencia de masa de los sedimentos. Por otro lado, la fuerte anomalía positiva de FAGA en el interior de la Cuenca Colombia este posiblemente asociado al adelgazamiento de la placa Caribe. Este trabajo también corrobora al igual que otros artículos la descompensación isostática de la Sierra Nevada de Santa Marta a partir de la anomalía positiva de FAGA, CBA y la correlación FAGA - peak to TGE - peak a partir de los índices locales de favorabilidad.

Los índices locales de favorabilidad permiten mostrar las correlaciones con mayor claridad, teniendo como ventaja la cuantificación de los datos, suministrando criterios para localizar áreas para prospección mineral y depósitos de hidrocarburo. Como se demostró en este trabajo, la correlación de FAGA y TGE permite mostrar el área y la extensión de las subcuencas de Tayrona, Urabá, Plato y San Jorge, siendo estas de gran interés exploratorio.

Para el abanico del Río Magdalena las anomalías de FAGA son de -20 a $-30 \mathrm{mGal}$ con un coeficiente de correlación espectral de -0,2548. Estos resultados permiten deducir un estado de sobrecompensación isostática, y como consecuencia un rebote isostático.

Finalmente, el análisis tectónico del noroccidente colombiano de acuerdo a los modelos 2D a partir de campos potenciales realizados en este trabajo, muestran un complejo arreglo tectónico el cual involucra un posible bajo ángulo de subducción de la placa Caribe $\left(\sim 10^{\circ}\right)$ debajo de la placa suramericana y la compleja disposición de los cinturones plegados de Sinú - San Jacinto.

Los espesores de la corteza continental se ven afectados por el bajo ángulo de la placa Caribe indicando espesores que varían entre 30 a $35 \mathrm{~km}$. Además, los modelos permiten también estimar un espesor promedio para la placa Caribe de $10 \mathrm{~km}$.

\section{AGRADECIMIENTOS}

Los autores agradecen al Departamento de Geociencias, Facultad de Ciencias de la Universidad Nacional de Colombia por los datos que dispone.

\section{REFERENCIAS}

Hernandez, O. (2006). Tectonic analysis of northwestern South America from integrated satellite, airborne and surface potential field anomalies. Ph.D. Thesis. The Ohio State University, USA.

Hinze, W., von Frese, R., and Saad, A. (2013). Gravity and magnetic exploration: Principles, practices and applications. Cambridge: Cambridge University Press.

Kearey, P., Klepeis, K.A., and Vine, F.J. (2009). Global tectonics. 3rd edition. New York: WileyBlackwell.

Kolla, V., Buffler, R.T., and Ladd, J.W. (1984). Seismic stratigraphy and sedimentation of Magdalena Fan, Southern Colombian Basin, Caribbean Sea. American Association of Petroleum Geologists Bulletin, 68(3), 316-332. 
Merriam, D.F., and Sneathh, P.H. (1966). Quantitative comparison of contour maps. Journal of Geophysical Research, 7(4), 1105-1115. doi: 10.1029/JZ071i004p01105.

Pince, J.M., Osorio, C., Mouly, B., and Amaral, J. (2003). Tertiary depositional environments and reservoir properties in the Sinu accretionary prism (offshore - Colombia). VIII Simposio Bolivariano - Exploración Petrolera en las Cuencas Subandinas. Cartagena, Colombia.

Pirmez, C., Breen, N.A., Flood, R.D., O’Connell, S., Jacobi, R.D., Ladd, J.W., Westbrook, G., Franco, J.V., and Garzon, M. (1990). Gloria mosaic of the Magdalena deep-sea fan, Northern Colombian convergent margin. American Association of Petroleum Geologists, 74(5), 741.

Romero-Otero, G., Slatt, R., and Pirmez, C. (2015). Evolution of the Magdalena deepwater fan in a tectonically active setting, Offshore Colombia. In: C. Bartilini, and P. Mann (Ed.). Petroleum geology and potential of the Colombian Caribbean Margin (pp. 675-708). Vol. 108. Texas: American Association of Petroleum Geologists.

von Frese, R.R., Jones, M., Kim, J., and Kim, J. (1997). Analysis of anomaly correlations. Geophysics, 62(1), 342-351. doi: 10.1190/1.1444136.

Watts, A.B. (2001). Isostasy and flexure of the lithosphere. 1st edition. New York: Cambridge University Press.

Luis Alejandro Garzón-Moreno

ORCID: 0000-0001-5182-3806

Orlando Hernández-Pardo

ORCID: 0000-0002-1297-8159

Trabajo recibido: noviembre 17 de 2017

Trabajo aceptado:mayo 09 de 2018 